\title{
Secular Increase in the Earth's LOD Strongly Implies that the Earth Might Be Expanding Radially on a Global Scale
}

\author{
Golden Gadzirayi Nyambuya \\ Department of Applied Physics, National University of Science and Technology, Bulawayo, \\ Republic of Zimbabwe \\ Email: physicist.ggn@gmail.com
}

Received 18 December 2013; revised 15 January 2014; accepted 23 January 2014

Copyright (C) 2014 by author and Scientific Research Publishing Inc.

This work is licensed under the Creative Commons Attribution International License (CC BY). http://creativecommons.org/licenses/by/4.0/

(c) ()

\begin{abstract}
Exactly 101 years ago, German scientist-Alfred Lothar Wegener, sailed against the prevailing wisdom of his day when he posited that not only have the Earth's continental plates receded from each other over the course of the Earth's history, but that they are currently in a state of motion relative to one another. To explain this, Wegener set forth the hypothesis that the Earth must be expanding as a whole. Wegener's inability to provide an adequate explanation of the forces and energy source responsible for continental drift and the prevailing belief that the Earth was a rigid solid body resulted in the acrimonious dismissal of his theories. Today, that the continents are receding from each other is no longer a point of debate but a sacrosanct pillar of modern geology and geophysics. What is debatable is the energy source driving this phenomenon. An expanding Earth hypothesis is currently an idea that is not accepted on a general consensus level. Antiproponent of the expanding Earth mercilessly dismiss it as a pseudo or fringe science with their main point of rejection being the energy source to power this supposed expansion. Be that as it may, we show herein that from the well accepted law of conversation of spin angular momentum, Stephenson [1]'s result that over the last 2700 years or so, the length of the Earth's day has undergone a change of about $+17.00 \mu \mathrm{s} / \mathrm{yr}$, this result invariably leads to the plausibility the Earth may very be expanding radially at a paltry rate of about $+0.60 \mathrm{~mm} / \mathrm{yr}$. If correct, this simple fact, automatically move the expanding Earth hypothesis from the realm of pseudo or fringe science, to that of plausible science.
\end{abstract}

\section{Keywords}

Astrometry; Celestial Mechanics; Ephemerides; Planets and Satellites; Formation 


\section{Introduction}

Exactly 101 years ago, German scientist-Alfred Lothar Wegener (1880-1930), sailed against the prevailing wisdom of his day when he posited that not only have the Earth's continental plates receded from each other over the course of the Earth's history, but that they are currently in a state of motion relative to one another [2] [3]. To explain this, Wegener set forth the hypothesis that the Earth must be expanding as a whole. Wegener's inability to provide an adequate explanation of the forces and energy source responsible for continental drift and the prevailing belief that the Earth was a rigid solid body resulted in the acrimonious dismissal of his theories. Today, that the continents are receding from each other is no longer a point of debate but a sacrosanct pillar of modern geology and geophysics. What is debatable is the energy source driving this phenomenon.

An expanding Earth hypothesis is currently an idea that is not accepted on a general consensus level. Foremost anti-proponents of the expanding Earth who hold the mainstream view in the field of plate tectonics, mercilessly dismiss (perhaps with good reasons) the Expanding Earth Hypothesis (EEH) as a pseudo or fringe science. This letter brings before the "eyes" of these anti-proponent hard-to-dismiss evidence that the Earth may very well be expanding or that a basis for this expansion has credible grounds. To the proponents of the EEH, we bring a reason to believe that physical evidence exists not only that points to the plausibility of EEH, but this expansion's plausibility is necessitated on the physical requirements needed to preserve the sacrosanct and embellished Laws of Physics such as the conservation of spin angular momentum.

The energy source of the expansion of the Earth is not thermal or emergent from any other mechanism other than the unlocking of the bound gravitational energy. Since the spin is dependant on three parameters, mass, radius or object and the spin period, the Laws of Physics vis the conservation of spin, requires that a change in the spin period, should automatically lead to a change in the radius of the object if the mass of the objects where to remain constant.

\section{Secular Increase in the LOD and the Expansion of the Earth}

Most-if not all, scientists (and as-well the non-scientists) with a sufficient or modicum of understanding of physics and physics principles will agree or attest to the fact that in the absence of interaction between orbital angular momentum $(\mathcal{J})$ and spin $(\mathcal{S})$, the angular momentum and spin will be conserved separately, that is $\dot{\mathcal{L}}=\dot{\mathcal{S}}=0$. In the event that there exists some spin-orbit interaction, then, the total angular momentum $\mathcal{L}=\mathcal{J}+\mathcal{S}$ is what must be conserved, that is $\dot{\mathcal{L}}=0$ such that $\dot{\mathcal{J}} \neq 0$ and $\dot{\mathcal{S}} \neq 0$. In the reading [4], we consider the option of spin-orbit interaction, where we concluded that the observed recession of the Earth- Moon system from the Sun must lead to the Earth expanding and the Moon to shrink. In the present letter, for the sack of the skeptic that might deny spin-orbit interaction, we drop this hypothesis of spin-orbit interaction and consider the scenario where spin and orbital angular momentum are conserved separately. The spin $\mathcal{S}=|\mathcal{S}|$ is a function of the mass $\mathcal{M}$, radius $\mathcal{R}$ and period $\mathcal{T}$ of the spinning object, that is $\mathcal{S}=2 \pi \mathcal{M} \mathcal{R}^{2} / \mathcal{T}$. Conservation of spin i.e. $\dot{\mathcal{S}}=0$ implies that $\dot{\mathcal{M}} / \mathcal{M}+2 \dot{\mathcal{R}} / \mathcal{R}-\dot{\mathcal{T}} / \dot{\mathcal{T}}=0$.

Now, if $\delta$ is to represent secular changes, then, the very fact that $\dot{\mathcal{S}}=0$ invariably implies that $\delta \mathcal{S}=0$, which in-turn implies that $\delta \mathcal{M} / \mathcal{M}+2 \delta \mathcal{R} / \mathcal{R}-\delta \mathcal{T} / \mathcal{T}=0$. For the Earth, we will have:

$$
\frac{\delta \mathcal{R}_{\oplus}}{\mathcal{R}_{\oplus}}=\frac{1}{2} \frac{\delta \mathcal{T}_{\oplus}}{\mathcal{T}_{\oplus}}-\frac{1}{2} \frac{\delta \mathcal{M}_{\oplus}}{\mathcal{M}_{\oplus}},
$$

where $\mathcal{M}_{\oplus}, \mathcal{R}_{\oplus}$ and $\mathcal{T}_{\oplus}$ are the mass, radius and period of the spin for the Earth respectively. From (1), in the absence of a secular variation in $\mathcal{T}_{\oplus}$, i.e. $\delta \mathcal{T}_{\oplus}=0$, an accreting Earth will lead to the Earth shrinking. On the other hand, an Earth with a non-varying mass will only expand if and only if there is a secular change in the Length of the Day (LOD). Such evidence exists!

In a remarkable study, Stephenson [5]; Stephenson and Morrison [1] have produced their seminal and landmarking works in the field of Earth's rotation spanning nearly three millennia i.e. from 700BC to 1990AD = 2690 years, where they concluded that the LOD is undergoing a secular variation of about $+17.00 \pm 5.00 \mu \mathrm{s} / \mathrm{yr}$. To come up with this remarkable conclusion, they have identified hundreds of eclipses and occultation observations in early European, Middle Eastern and Chinese annals, manuscripts, canons and records. Of these data, since the dawn of humankind, in spite of their relatively low precision, these data represent humanity's only record of the variation of the LOD over a lengthy period of time. 
Given that the present mean LOD $\mathcal{T}_{\oplus}=23.93 \mathrm{hr}$ it follows that the Earth's radius may not only be changing, but increasing, i.e.:

$$
\delta \mathcal{R}_{\oplus}=+0.60 \pm 0.10 \mathrm{~mm} / \mathrm{yr} .
$$

In the reading [4] where the spin-orbit interaction and the recession of the Earth-Moon system is taken into account, one finds an expansion rate of about $+1.50 \mathrm{~mm} / \mathrm{yr}$ which is-at least, about twice that obtained above. The difference can safely be attributed to the here unaccounted for phenomenon of spin-orbit interaction.

\section{Hubble Expansion of the Earth}

If the Earth is expanding radially as a whole, then, the very surface of the Earth would expand as neighbouring points drift apart in the literal directions. If this where true, then the very ground making up the Earth would open up slowly and evidence of this would be so evident as one would see trenches on the surface of the Earth. No such global phenomenon is known in the history of the Earth nor do we see trenches all over the globe. Certainly, this is not the kind of expansion we envisage. We envisage-as shown in the diagram below; the Earth's crust sitting on an expanding upper mantle where the Earth expands radially from the center.

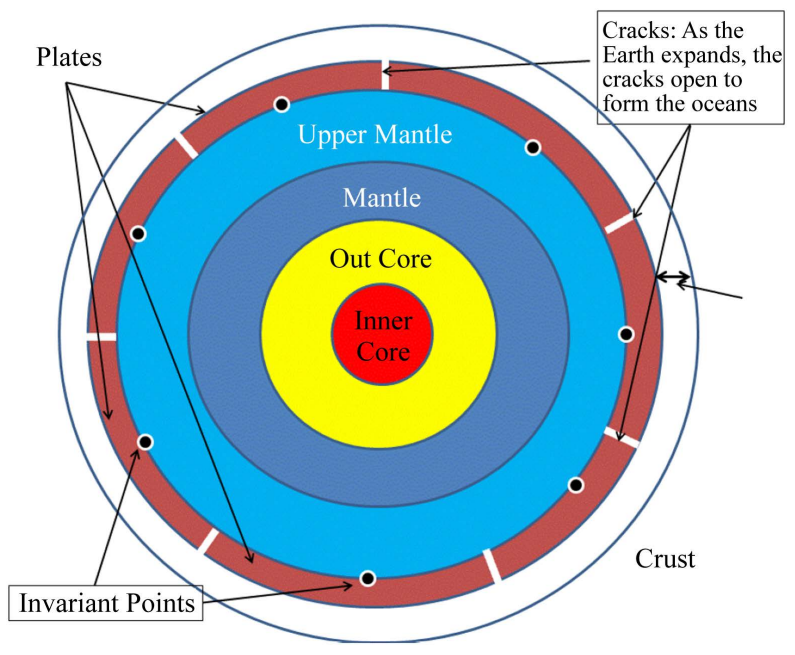

Initially-i.e., when the Earth began its hypothetical expansion, we must have had water covering the entire Earth crust which itself [crust] was sitting on-top of a molten upper mantle. As the Earth began to expand from its center radially outward up-till the upper mantle, the crust developed cracks. These cracks separated the different lands thus formed the various plates that make the Earth today. The spaces that opened up as the cracks widened must have gone on-to form the oceans we see today.

As the crust sits on the expanding Earth, the created plates may exhibit some rotation due to the mantle currents. Be that it may, there are points on the mantle that will act as the centre of the plate and these points will not take part in the rotation. These points are what we have labelled as invariant points. In general, we can take any two points that are an angular distance $\theta$ apart. Their distance, $D$ apart on the surface of the Earth is $D=\mathcal{R}_{\oplus} \theta$, differenting this with respect to time and then dividing with the same expression, we will have:

$$
\frac{\dot{D}}{D}=\frac{\dot{\theta}}{\theta}+\frac{\dot{\mathcal{R}}_{\oplus}}{\mathcal{R}_{\oplus}},
$$

For the invariant points $\dot{\theta}=0$, so that:

$$
\dot{D}=\left(\frac{\dot{\mathcal{R}}_{\oplus}}{\mathcal{R}_{\oplus}}\right) D,
$$

which is a Hubble-type expansion. If the Earth really is expanding, then, if one calculated the drift with respect to other points on different plates of a choose point on some plate, then, if they plotted a graph of $\dot{D}$ vs $D$, 
they are likely to get a graph with scattered points and this scattering occurs because $\dot{\theta} \neq 0$ and $\dot{\theta} / \theta$ is certainly not uniform, hence the scatter of points. We know there are mantle currents and these currents are what will give raise to the scatter. However, if one where to search for invariant points on each plate and calculated the drift of other invariant points relative to a choose invariant point on a given plate, then, they are most likely going to get a linear graph should they plot a graph of $\dot{D}$ vs $D$. Using Earth motion calculators, this idea is currently being worked-on i.e., the idea of finding invariant points and testing the radially EEH.

\section{Measurements Using VLBI, LLR, SLR, and GPS Techniques}

Measurements obtained from the latest, modern and most precise Very Long Baseline Interferometry (VLBI), Lunar Laser Ranging (LLR), Satellite Laser Ranging (SLR), and Global Positioning System (GPS) techniques have yielded results that strongly appear to suggest that the Earth may not be undergoing any significant and noticeable radial expansion. This has led to a general chorus amongst the advocates of the anti-expansion of the Earth to conclude that there is no further evidence needed-the Earth is simple not undergoing any expansion. Our feeling about these measurements is that they most certainly put an upper limit to the expansion itself. Further we believe there is need to understand how the gravitational field of an expanding body interacts with these measuring instruments before dismissing the idea of an exanding earth.

\section{Plausible Initial Earth Radius}

If we assumed that the Earth's landmass was once beneath the waters that once filled all of the Earth and that this landmass emerged above the surface of the Earth as a result of the expansion of the Earth, then, given that the current landmass is $\sim 29.1 \%$ of the total surface area of the Earth, it follows that the initial radius of the Earth $\mathcal{R}_{\oplus}(0)$ must have been about one half the present Earth radius $\mathcal{R}_{\oplus}$, i.e.:

$$
\mathcal{R}_{\oplus}(0)=0.539 \mathcal{R}_{\oplus}=3.45 \times 10^{6} \mathrm{~m} .
$$

The above calculation assumes that the current landmass was once one landmass making up a sphere and this landmass did not increase in surface area but seat on an expanding lithosphere in which process it emerged above the waters and these water filled the emerging spaces in-between. Assuming the nominal radial expansion rate of $+0.60 \mathrm{~mm} / \mathrm{yr}$, (5) would mean that the Earth must be $\sim 4.90 \times 10^{9} \mathrm{yrs}$ old. This age is significantly above $(\sim 17 \%)$ the measured age of the Earth which is assumed (via radioactive measurements and other means) to be about $4.20 \times 10^{9}$ yrs .

On the other hand, if we assume the Earth is about $4.20 \times 10^{9} \mathrm{yr}$ and that it has been expanding steadily at the nominal rate of $\sim+0.60 \mathrm{~mm} / \mathrm{yr}$, then, $\mathcal{R}_{\oplus}(0)=0.606 \mathcal{R}_{\oplus}=3.88 \times 10^{6} \mathrm{~m}$. In both calculations conducted above, we get reasonable agreement between the two somewhat independent calculations-this is a good sign. Crudely, it appears reasonable from the foregoing to imagine that the Earth may have grown from an initial Earth that was about one-half the current radius of the Earth.

\section{Discussion and Conclusion}

Perhaps, with good reason, the anti-proponents of the EEH have been right to vehemently dismiss the EEH as fringe and or pseudo-science for the simple reason that the proponents of the EEH have in most cases presented far from home solutions for the mechanism that might drive this expansion. For example, Dr. Hugh Owen suggested in his book, Atlas of Continental Displacement, 200 Million Years to the Present: A Test of the Conventional and Expanding Earth Models, that there was a phase change in the Earth's core that produced a volume change in the Earth while the mass stayed the same [6]. It is difficult if not impossible to verify this claim/hypothesis.

Another favourite mechanism for the EEH is that the mass of the Earth must be increasing. That the mass of the Earth is increasing is not wrong at all, it is correct. The Earth's mass increases due to radiation it absorbs from the Sun. Further, the Earth is under the constant bombardment of cosmic showers (meteorites, cosmic rays, etc), these obviously add small amounts of material over short periods. The proponents of the EEH then say, "but over geological times ... these cosmic showers become 'cosmic storms', leading to a significant increase in the Earth's radius". We did argue that an increase in the mass of the Earth must instead lead to a shrinking and not expansion of the Earth. Besides, the amount of mass increase of the Earth is so small that it should not 
lead to a significant change in the radius of the Earth. The required secular mass loss rate of the Earth $\delta \mathcal{M}_{\oplus} / \mathcal{M}_{\oplus}$, must be of the order of $\sim 1.00 \times 10^{-10} \mathrm{yr}^{-1}$ if at all this were to lead to a significant change in the Earth's radius of the order of $\sim+1.00 \mathrm{~mm} / \mathrm{yr}$, at any rate, this is a very high unacceptable mass loss rate.

On the list of the EEH, there is one notable present day advocate perhaps worthy of mention i.e., America's interdisciplinary scientist Dr. J. Marvin Herndon. He has setforth what he calls "Whole-Earth Decompression Dynamics" (WEDD), which he describes as a theory combining elements of plate tectonics and Earth expansion [7]. This WEDD theory holds that Earth formed from a Jupiter-sized gas giant by catastrophic loss of its gaseous atmosphere with subsequent decompression and expansion of the rocky remnant planet resulting in decompression cracks at continental margins which are filled in by basalts from mid-ocean ridges. Despite it being physically possible, this hypothesis is very difficult to prove, let alone believe. Anti-proponents of the EEH will obviously have a field day in dressing-down such a hypothesis on the basis of lack of evidence to prove its viability.

Surely, insofar as explaining very well many facts about our present World, the idea of an expanding Earth has been shown to work [8]-[10]. However, in science, that is not a good enough reason to believe the Earth must be expanding. It is only a good enough reason to set the hypothesis that the Earth might be expanding; nothing more and nothing less. Thereafter, one must now go onto seek ponderable evidence in support or against the hypothesis. The acceptance of a hypothesis as fact is similar or akin to String Theory ${ }^{1}$ whose exquisite mathematical beauty, elegance and consistency with Einstein's General Theory of Relativity (GTR) and Quantum Mechanics (QM), has persuaded many to come so close as to declaring this yet-to-be-tested theory a fact of experience without it submitting to the rigorous yardsticks of science that would place this theory on a pedestal and correspondence with physical experience. For as long as we are still doing science, there is always the need to come up with as close to home as is possible solutions or theories (that are readily falsifiable) which in the present case these are theories as to why the Earth is or must be expanding and these theories must easily be verified or refuted by observations and experience.

Logically, there are two obvious possibilities, (1) either the Earth is expanding or, (2) the Earth is not expanding. One of the two of these possibilities must be true and verifiable to within reasonable, believable and acceptable accuracy. We believe the simple ideas laid down herein and as-well as in the reading [4] are close to home solutions to the problem of the EEH and these solutions are based on solid observational evidence in the form of (1) the variation in the LOD and (2) the observed recession of the Earth-Moon system from the Sun. These pieces of evidence can not be dismissed so easily, one is involuntarily forced somehow to consider for a minute or so, that yes, the Earth must be expanding somehow. At our disposal, the recession of the Earth-Moon system from the Sun is the most indirect piece of evidence yet, that, the Earth must indeed be expanding if at all the Laws of Physics are to hold as most would believe they do hold at all times i.e., yesterday, now and forever. Off cause, for whatever reason, one may not want the Earth to expand, but sadly and against our foremost desideratum, Nature does not at all caterer for our feelings or wishes in this regard or on this front; facts are facts, they are hard to dismiss, let alone-ignore.

Despite its abysmal dismissal by the mainstream researcher(s), the EEH has or is supported by a number of credible authors (e.g. [11]-[17], amongst many others) who have surely contributed significantly to the field of knowledge of geology, geophysics and science in general. The only problem in getting this hypothesis being accepted by the mainstream is the completion of the hypothesis by obtaining a credible source of energy for the supposed or hypothesised expansion. Clearly, as suggested in the reading [4], a credible source of energy driving this phenomenon is the orbital kinetic energy of the Earth-Moon system that is converted to kinetic energy associated with the spin of the spinning object. This same energy is what must drive the secular change in the LOD. Changes in the spin affect the spin period and as-well the spatial size of the spinning object -- hence the Earth must either expand or contract, the situation on the ground is that the Earth must expand.

In a nutshell, what the present letter has sought to furnish is that in the case that one does not believe or does not want to believe at all in the possibility that the Earth might be or has expanded since it came into being, we have presented the remarkable findings of Stephenson [5]; Stephenson and Morrison [1] so that they may or might make the endeavour to comprehend these findings in the light of the conservation of spin angular momentum. If at all they do comprehend this well enough, then, we are left in no doubt whatsoever that they will

\footnotetext{
${ }^{1}$ String Theory is a theory considered-by a significant number of researchers; to be the most promising theory that seeks science's greatest and foremost endeavour of unifying all of the forces of Nature (i.e., the Gravitational, Electromagnetic, Weak, and Strong nuclear forces) into one consistent mathematical framework and physical theory.
} 
without fail accept that the Earth must be expanding, at least by a magnitude of about $+0.60 \mathrm{~mm} / \mathrm{yr}$. In summary, all we have done herein, is to set aside the issue of spin-orbit interaction, in case this is used as a weapon to dressing-down the idea that the Earth must be expanding.

In closing, though we await solid confirmation from observations and experience, if we are to express our uttermost confidence (and not faith) in the Laws of Physics, we do not have to say "The Earth might be expanding globally" but that "The Earth must be expanding globally" at a rate of at least $+0.60 \mathrm{~mm} / \mathrm{yr}$.

\section{Conclusion}

Assuming the correctness or the acceptability of the thesis put forward herein, we hereby make the following conclusion that if one assumes the Earth to be an isolated system (of which it is not), the current secular change in the LOD deduced by Stephenson [5]; Stephenson and Morrison [1] of $17.00 \pm 5.00 \mu \mathrm{s} / \mathrm{yr}$, then, the Earth may very well be expanding radially on a global scale at a steady rate of at least $+0.60 \mathrm{~mm} / \mathrm{yr}$.

\section{References}

[1] Stephenson, F.R. and Morrison, L.V. (1995) Long-Term Fluctuations in the Earth's Rotation: 700 BC to AD 1990. Philosophical Transactions of the Royal Society A, 351, 165-202. http://dx.doi.org/10.1098/rsta.1995.0028

[2] Wegener, A.L. (1912) Die Entstehung der Kontinente. Geologische Rundschau, 3, 276-292. http://dx.doi.org/10.1007/BF02202896

[3] Wegener, A.L. (1912) Die Entstehung der Kontinente. Petermann’s Geographische Mitteilungen, 58, 185-195, 253256, 305-308.

[4] Nyambuya, G.G. (2014) On the Expanding Earth and Shrinking Moon. International Journal for Astronomy and Astrophysics, 4, 227-243.

[5] Stephenson, F.R. (1997) Historical Eclipses and Earth’s Rotation. Cambridge University Press, Cambridge. http://dx.doi.org/10.1017/CBO9780511525186

[6] Owen, H. (1983) Atlas of Continental Displacement, 200 Million Years to the Present (Cambridge Earth Science Series). Cambridge University Press, Cambridge.

[7] Herndon, J.M. (2005) Whole-Earth Decompression Dynamics. Current Science, 89, 10.

[8] Romm, J. (1994) A New Forerunner for Continental Drift. Nature, 367, 407-408. http://dx.doi.org/10.1038/367407a0

[9] Mantovani, R. (1909) L'antarctide. Je minstruis. La Scieence Pour Tous, 38, 595-597.

[10] Mantovani, R. (1889) Les Fractures de Lécorce Terrestre et la Théorie de Laplace. Bulletin de la Société des Sciences et Arts de l'Ile de la Réunion, 41-53.

[11] Carey, S.W. (1975) The Expanding Earth—An Essay Review. Earth Science Reviews, 11, 105-143. http://dx.doi.org/10.1016/0012-8252(75)90097-5

[12] Carey, S.W. (1970) Australia, New Guinea and Melanes in the Current Revolution in Concepts of the Evolution of the Earth. Search, 1, 178-189.

[13] Creer, K.M. (1965) An Expanding Earth. Nature, 205, 539-544. http://dx.doi.org/10.1038/205539a0

[14] Ward, M.A. (1963) On Detecting Changes in the Earth’s Radius. Geophysical Journal of the Royal Astronomical Society, 8, 217-225. http://dx.doi.org/10.1111/j.1365-246X.1963.tb06285.x

[15] Cox, A. and Doell, V.R. (1961) Paleomagnetic Evidence Relevant to a Change in the Earth's Radius. Nature, $189,45$. http://dx.doi.org/10.1038/189045a0

[16] Egyed, L. (1961) Palaeomagnetism and the Ancient Radii of the Earth. Nature, 190, 1097-1098. http://dx.doi.org/10.1038/1901097a0

[17] Heezen, B.C. (1960) The Rift in the Ocean Floor. Scientific American, 203, 98-110. http://dx.doi.org/10.1038/scientificamerican1060-98 